\title{
Extensive calcific myonecrosis of the lower leg treated with free tissue transfer
}

\author{
Tae Gon Kim, Yong Sakong, Il Kug Kim \\ Department of Plastic and Reconstructive Surgery, Yeungnam University College of Medicine, Daegu, Korea
}

\begin{abstract}
Calcific myonecrosis is a rare condition in which hypoperfusion due to compartment syndrome causes soft tissue and muscle to become calcified. As calcific myonecrosis gradually deteriorates, secretions steadily accumulate inside the affected area, forming a cavity that is vulnerable to infection. Most such cases progress to chronic wounds that are unlikely to heal spontaneously. After removing the calcified tissue, the wound can be treated by primary closure, flap coverage, or a skin graft. In this case, a 72-year-old man had extensive calcific myonecrosis on his left lower leg, and experienced swelling and increasing tenderness. After removing the muscle calcification, we combined two anterolateral thigh free flaps, which were harvested from the patient's right and left thigh, respectively, to reconstruct the wound with a dead-space filler and skin-defect cover at the same time. The patient recovered without revision surgery or major complications.
\end{abstract}

Keywords Calcific myonecrosis / Compartment syndrome / Trauma / Lower limb / Free flap

\author{
Correspondence: Tae Gon Kim \\ Department of Plastic and \\ Reconstructive Surgery, Yeungnam \\ University College of Medicine, 170 \\ Hyeonchung-ro, Nam-gu, Daegu \\ 42415, Korea 03722, Korea \\ Tel: +82-53-626-3480 \\ Fax: +82-53-626-0705 \\ E-mail:kimtg0919@daum.net
}

Received: June 25, $2020 \bullet$ Revised: July 28, $2020 \bullet$ Accepted: September 23, 2020

plSSN: 2234-6163 • elSSN: 2234-6171 • https://doi.org/10.5999/aps.2020.01200 • Arch Plast Surg 2021;48:329-332

\section{INTRODUCTION}

Calcific myonecrosis is a rare condition in which soft tissue and muscle become calcified. The exact cause is unknown, although a probable mechanism is calcification after necrosis and fibrosis of soft tissue and muscle due to ischemia of the lower limbs after compartment syndrome. As the condition progresses, the cavity expands and fluid accumulates. If an open wound is also present, an infection can occur. The cavity may gradually increase in size and turbid discharge may accumulate, resulting in a chronic wound [1]. Proper wound management is essential, as it is difficult for such a wound to heal naturally. Traditionally, these are considered "no-touch lesions" because once an infection occurs, it is difficult to manage and the condition can progress extensively. Amputation may be required in some cases [1]. However, in recent years, successful treatment of patients with calcific myonecrosis has been reported due to advances in wound treatment and flap surgery. In this case report, we describe a patient with extensive calcific myonecrosis lesions of the lower extremity along with an open wound and infection that was treated using serial debridement and a combined free flap. We obtained the approval of the Yeungnam University Hospital Institutional Review Board (IRB No. 2020-05-045) and the patient's consent for this case study.

\section{CASE}

A 72-year-old man visited our hospital with a painless mass on lateral side of his left lower limb that had developed 4 years previously. He had undergone surgery on his left lower limb twice ( 25 and 55 years beforehand). The patient had a history of hypertension, diabetes, and benign prostatic hyperplasia. A biopsy 
performed at the orthopedic department showed calcification of the soft tissue. X-rays of the left lower extremity showed extensive calcification, which included the anterior and lateral compartments and a portion of the posterior compartment (Fig. 1).

The patient presented to the hospital again with swelling and tenderness at the biopsy site. On examination, an open wound was observed on the anterolateral side of the proximal left lower leg, and a cavity had formed from which a discharge emerged. The turbid secretion contained calcium debris. The cavity extended to just above the ankle. An incision was made extending to the ankle, and debridement and irrigation were performed, after which a betadine-soaked gauze dressing was applied (Fig. 2). Methicillin-resistant Staphylococcus aureus (MRSA) was identified in the wound culture, and vancomycin was intravenously administered. Osteomyelitis was not confirmed in the threephase bone scan test.

After continuous dressings were applied, the discharge and debris started to reduce. The patient had an open wound on the left lower leg measuring $17 \times 4 \mathrm{~cm}^{2}$. The tibia was exposed inside the wound, and focal granulation tissue had formed on the
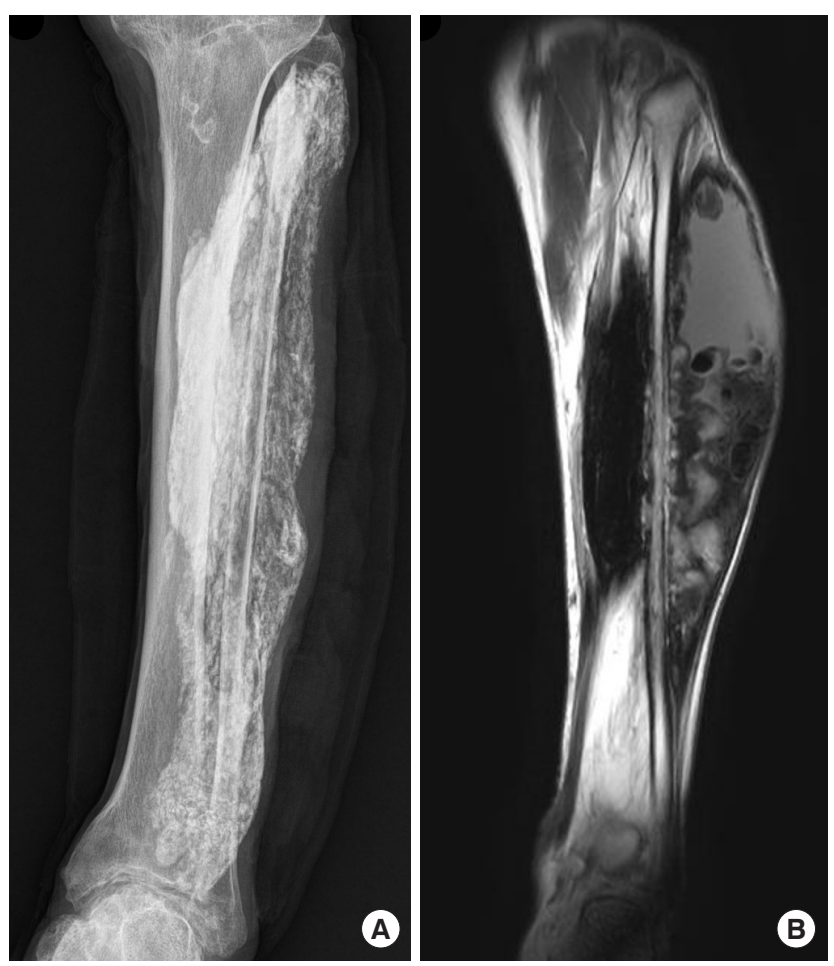

Fig. 1. Radiographs and magnetic resonance imaging findings. (A) Anteroposterior radiographs of the left lower leg showing extensive calcification of the tibialis anterior muscle, extensor hallucis longus muscle, extensor digitorum longus muscle, peroneus brevis muscle, peroneus longus muscle, and focal flexor hallucis longus muscle. (B) T2-weighted coronal magnetic resonance imaging view showing fluid collection with an intense signal in the upper area of the calcified muscles. bony surface. Negative-pressure wound therapy (NPWT) was applied to reduce the size of the wound. However, primary closure was not possible due to the large defect site and the slow growth of granulation tissue. Free flap coverage was planned once MRSA was no longer found in the wound culture and the calcium debris was reduced during irrigation. A hand-held Doppler test and three-dimensional angiography showed that the left anterior tibial artery displayed poor perfusion, but the posterior tibial artery and peroneal artery were intact.
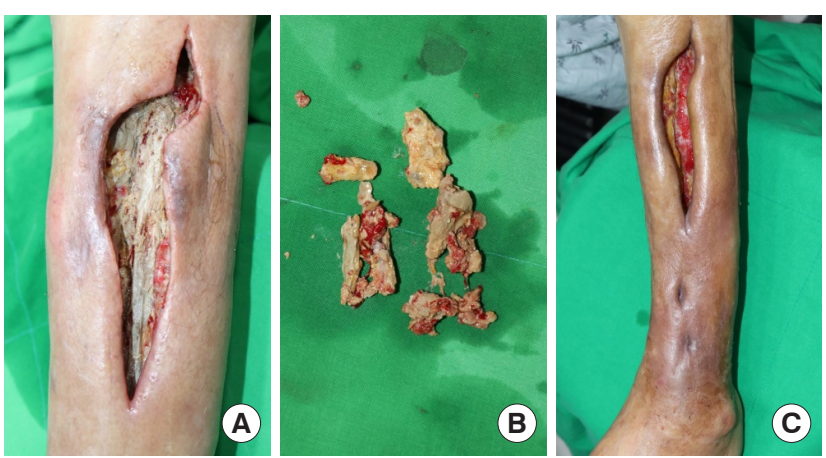

Fig. 2. Clinical photographs of the leg after biopsy. (A) Open wound with pocket formation after biopsy, and the incision for irrigation and debridement of the calcification. (B) Calcific debris was removed. Daily irrigation and debridement were performed. (C) Preoperative clinical photograph immediately before flap surgery. The calcified soft tissue had been removed from most of the wound base and granulation was in progress over the tibial bone.

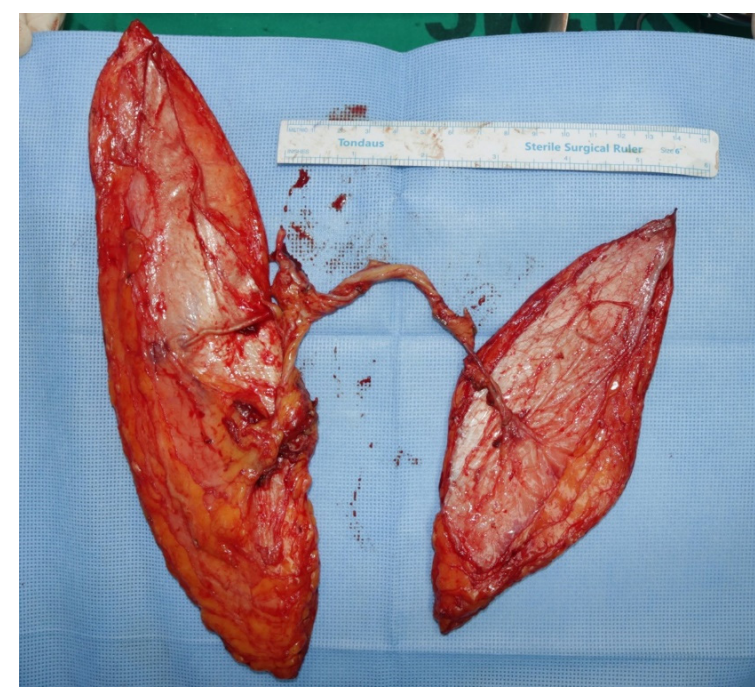

Fig. 3. Clinical photographs of intraoperative findings. The left thigh free flap (right side) measured $25 \times 7 \mathrm{~cm}^{2}$ and the right thigh free flap (left side) measured $18 \times 8 \mathrm{~cm}^{2}$. The pedicle of the right thigh free flap was connected to the descending branch of the lateral circumflex artery of the left thigh. The combined flap was then harvested. The right thigh flap was de-epithelialized and positioned inside the cavity. 

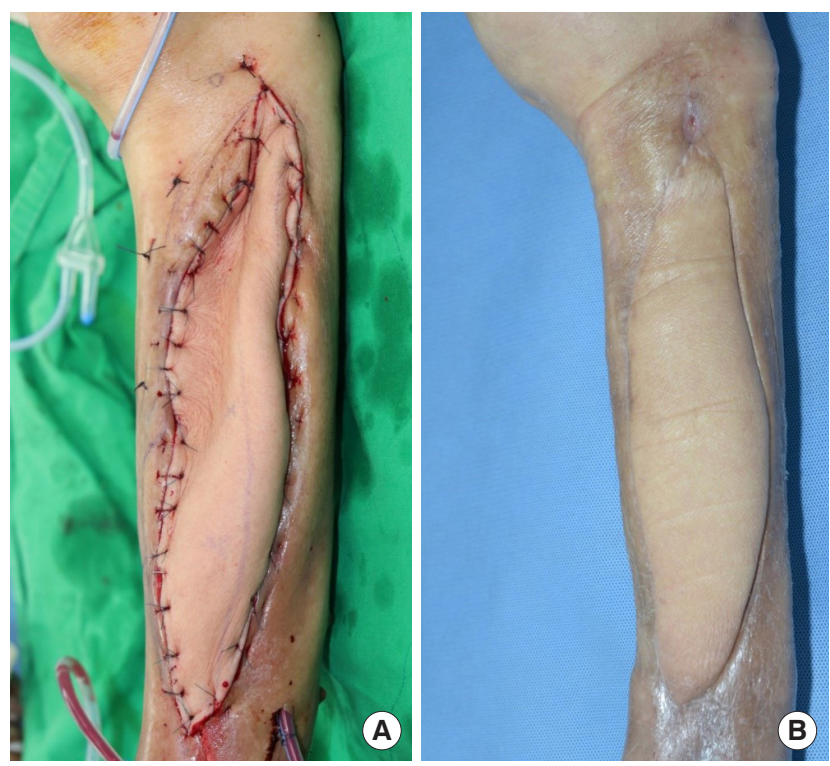

Fig. 4. Clinical photographs of postoperative findings. (A) The external free flap (left thigh) was sutured. (B) At 6 months after the operation, the wound has recovered without complications.

Two anterolateral thigh (ALT) free flaps were planned: an $18 \times 8 \mathrm{~cm}^{2}$ flap from the right thigh and a $25 \times 7 \mathrm{~cm}^{2}$ flap from the left thigh. The purpose of the right thigh flap was to fill the cavity, while the goal of the left thigh flap was to cover the defect. After lifting both ALT flaps, we connected the right flap pedicle to the distal portion of the descending branch of the lateral circumflex femoral artery of the left ALT free flap (Fig. 3). With the pedicle combined, the proximal portion of the descending branch was cut and anastomosed to the left posterior tibial vessel in an end-to-side manner for the artery and an end-to-end manner for the concomitant vein. The right ALT free flap was inset in the cavity after de-epithelization and then fixed with an absorbable suture. The left ALT was used to cover the defect. After surgery, the flap perfusion was excellent, and a drainage tube was inserted into the flap and donor sites. The patient was discharged 1 month after surgery. He remained well over a 12week follow-up period (Fig. 4). The surgical site did not become infected and the wound did not recur.

\section{DISCUSSION}

The pathophysiology of calcific myonecrosis has yet to be conclusively established. Blood supply failures due to compartment syndrome are thought to cause necrosis in the muscle compartment [1]. Intralesional hemorrhage and seroma collection increase the size of the mass, after which calcification occurs. The anterior and lateral compartments of the lower leg are generally affected, as well as the deep posterior compartment. During skeletal muscle debridement, dense fibrous connective tissue, including extensive myelinated nerve tissue, calcific deposits, and inflammatory cells are found [1-3]. Calcific myonecrosis is mainly diagnosed based on radiological findings. X-rays show distinctive calcification patterns depending on which lower leg compartment is involved, and contrast-enhanced computed tomography shows homogeneous density and a peripheral calcification rim around the mass. Various studies have reported that signal patterns on $\mathrm{T} 1$ and $\mathrm{T} 2$ magnetic resonance imaging differ according to the content of the muscle. If there is a liquid collection inside the mass, a homogeneous center is observed on T2 images. When calcification is also present, a heterogeneous mass or peripheral rim enhancement can be seen on $\mathrm{T} 1$ and $\mathrm{T} 2$ images [4-6]. Biopsy can be performed to differentiate calcific myonecrosis from other diseases. Nakayama et al. [7] reported that biopsy findings showed calcification and extensive necrotic tissue with focal inflammatory cell infiltration.

The differential diagnosis of calcific myonecrosis is a calcified soft-tissue mass (e.g., such as myositis ossificans), synovial sarcoma, epithelioid sarcoma, tumoral calcinosis, dermatomyositis, polymyositis, and posttraumatic pseudoaneurysm $[3,5,7,8]$. Myositis ossificans is a heterotopic nonneoplastic bone formation in soft tissue with self-limiting growth. Initially, calcification appears in the periphery of the lesion, and as the size increases, a round bone mass appears. Sarcomas generally manifest as nonperipheral lesions with calcifications, not limited to a single muscle group, and liquefaction in the center of calcified lesions is rare. In dermatomyositis, polymyositis, or diabetic osteonecrosis, calcification is extensive, but these conditions do not involve a history of trauma and are mostly accompanied by systemic symptoms.

There is no consensus on the optimal treatment for calcific myonecrosis. Although the lesion itself is benign, there is a high incidence of complications such as sinus tract formation and infection after excisional biopsy, surgical debridement, and resection [1]. If the lesion is small and asymptomatic, it may be acceptable to undertake careful observation of it over time. However, a surgical intervention is necessary when the lesion is too large for secondary healing or an infection occurs. After complete mass excision, primary closure, flap coverage, or a skin graft can be performed. Dead space can be reduced by tissue granulation using NPWT [9]. Jassal et al. [3] treated calcific myonecrosis with an abdominis muscle flap after surgical debridement, similar to this case.

In this case, extensive calcification myonecrosis occurred in the anterior part of the lower leg, which resulted in the formation of a massive cavity. After irrigation and surgical debridement, the amount of calcific debris decreased, and an attempt 
was made to induce granulation tissue growth using NPWT. However, this was not successful. In previous case reports, a musculocutaneous flap or a single free flap that was partly deepithelialized was used in the cavity $[3,10]$. In this case, it was challenging to restore the dead space with a single free flap. Therefore, a combined ALT free flap was performed to treat the cavity and skin defect simultaneously. For the combined flap to fill the dead space, a rectus abdominis muscle flap or latissimus dorsi flap could also be used. If a vessel injury or vessel calcification has been caused by compartment syndrome or trauma, it may be challenging to find the recipient vessel. Therefore, the patient's vascular condition should be evaluated using Doppler or three-dimensional angiography before surgery.

Calcific myonecrosis is a rare condition that most often occurs in the lower limbs after compartment syndrome. It can be diagnosed based on imaging findings and the patient's history. Conservative management is possible if sinus formation and discharge do not worsen. However, a large cavity or wound infection may require surgical debridement. Wound coverage can be achieved with various methods, including a local flap, NPWT, or a free flap. In cases with a large cavity and significant skin defect, a combined free flap can be efficacious.

\section{NOTES}

\section{Conflict of interest}

Tae Gon Kim is an editorial board member of the journal but was not involved in the peer reviewer selection, evaluation, or decision process of this article. No other potential conflicts of interest relevant to this article were reported.

\section{Ethical approval}

The study was approved by the Institutional Review Board of Yeungnam University Hospital (IRB No. 2020-05-045) and performed in accordance with the principles of the Declaration of Helsinki. Written informed consent was obtained.

\section{Patient consent}

The patient provided written informed consent for the publication and the use of his images.

\section{Author contribution}

Project administration: TG Kim. Writing-original draft: Y Sakong. Writing-review \& editing: TG Kim, IK Kim.

\section{ORCID}

Tae Gon Kim

Yong Sakong

Il Kug Kim

https://orcid.org/0000-0002-6738-4630

https://orcid.org/0000-0003-3185-5231

https://orcid.org/0000-0002-2428-8403

\section{REFERENCES}

1. O’Dwyer HM, Al-Nakshabandi NA, Al-Muzahmi K, et al. Calcific myonecrosis: keys to recognition and management. AJRAm J Roentgenol 2006; 187:W67-76.

2. O'Keefe RJ, O'Connell JX, Temple HT, et al. Calcific myonecrosis: a late sequela to compartment syndrome of the leg. Clin Orthop Relat Res 1995:205-13.

3. Jassal DS, Low M, Ross LL, et al. Calcific myonecrosis: case report and review. Ann Plast Surg 2001;46:174-7.

4. Okada A, Hatori M, Hosaka M, et al. Calcific myonecrosis and the role of imaging in the diagnosis: a case report. Ups J Med Sci 2009;114:178-83.

5. Zohman GL, Pierce J, Chapman MW, et al. Calcific myonecrosis mimicking an invasive soft-tissue neoplasm: a case report and review of the literature. J Bone Joint Surg Am 1998; 80:1193-7.

6. Tuncay IC, Demirors H, Isiklar ZU, et al. Calcific myonecrosis. Int Orthop 1999;23:68-70.

7. Nakayama D, Susa M, Horiuchi K, et al. Expansile calcific myonecrosis arising in the lower leg: a case report and recommendation for treatment. J Orthop Sci 2019 Dec 20 [Epub]. https://doi.org/10.1016/j.jos.2019.11.009.

8. Janzen DL, Connell DG, Vaisler BJ. Calcific myonecrosis of the calf manifesting as an enlarging soft-tissue mass: imaging features. AJR Am J Roentgenol 1993;160:1072-4.

9. Sreenivas T, Nandish Kumar KC, Menon J, et al. Calcific myonecrosis of the leg treated by debridement and limited access dressing. Int J Low Extrem Wounds 2013;12:44-9.

10. Ao M, Uno K, Maeta M, et al. De-epithelialised anterior (anterolateral and anteromedial) thigh flaps for dead space filling and contour correction in head and neck reconstruction. Br J Plast Surg 1999;52:261-7. 